\title{
WEAK MAXIMALITY CONDITION AND POLYCYCLIC GROUPS
}

\author{
Y. K. KIM AND A. H. RHEMTULLA
}

(Communicated by Ron Solomon)

\begin{abstract}
A group $G$ is called strongly restrained if there exists an integer $n$ such that $\left\langle x^{(y)}\right\rangle$ can be generated by $n$ elements for all $x, y$ in $G$. We show that a group $G$ is polycyclic-by-finite if and only if $G$ is a finitely generated strongly restrained group in which every nontrivial finitely generated subgroup has a nontrivial finite quotient. This provides a general setting for various results in soluble and residually finite groups that have appeared recently.
\end{abstract}

\section{INTRODUCTION}

We call a group $G$ restrained if $\left\langle x^{(y)}\right\rangle$ is finitely generated for all $x, y$ in $G$. And we call $G$ strongly restrained if there exists an integer $n$ such that $\left\langle x^{(y)}\right\rangle$ can be generated by $n$ elements for all $x, y$ in $G$. The purpose of looking at such groups is that they provide a general setting for various results in soluble and residually finite groups that have appeared recently.

Restrained groups contain periodic groups, Engel groups, and groups satisfying the maximal condition for subgroups locally. Strongly restrained groups contain groups of bounded exponent, bounded Engel groups, and collapsing groups. The notion of collapsing groups was introduced by Semple and Shalev in [5]. A group $G$ is $n$-collapsing if for any set $S$ of $n$ elements in $G$, $\left|S^{n}\right|<n^{n}$ and $G$ is a collapsing group if it is $n$-collapsing for some $n>0$.

The existence of Tarski monsters by Ol'shanskii [4] shows that strongly restrained groups (with $n=2$ ) can be wild! In order to avoid such groups, if we impose the condition that every nontrivial finitely generated subgroup of $G$ has a nontrivial finite quotient, then we obtain the following satisfactory description of strongly restrained groups.

Theorem A. A group $G$ is polycyclic-by-finite if and only if $G$ is a finitely generated strongly restrained group in which every nontrivial finitely generated subgroup has a nontrivial finite quotient.

In the rest of this paper we shall say $G$ is locally graded to mean that every finitely generated nontrivial subgroup of $G$ has a nontrivial finite quotient. The groups constructed by Gupta and Sidki in [3] are infinite, periodic, finitely

Received by the editors June 15, 1993.

1991 Mathematics Subject Classification. Primary 20F19; Secondary 20E25, 20 E26. 
generated, and residually finite. As periodic groups they are restrained, and residual finiteness implies that the groups are locally graded. Thus we cannot replace "strongly restrained" by "restrained" in Theorem A. However, it is not difficult to show that locally soluble restrained groups are locally polycyclic. Our method also allows us to show the following.

Theorem B. A finitely generated collapsing right orderable group is nilpotent-byfinite.

Recall that $G$ is right orderable (or an $R O$-group) if there is a total order relation $\leq$ on $G$ such that for all $a, b, x$ in $G, a \leq b$ implies $a x \leq b x$. A right order $\leq$ on $G$ can be characterized by its positive cone $P=\{g \in G ; e \leq$ $g$ \} which has the three properties: (i) $P P=P$; (ii) $P \cup P^{-1}=G$; and (iii) $P \cap P^{-1}=\{e\}$. A right order $\leq$ on $G$ is called a $C$-order if for each pair of elements $a, b$ in $P$ there exists some positive integer $m$ such that $a \leq a^{m} b$. It is known that an $R O$-group that has a $C$-order is locally indicable and hence locally graded. We show that every right order in a collapsing $R O$-group $G$ is a $C$-order.

\section{ProOFS}

We begin by showing that Engel groups and collapsing groups are restrained. This is then followed by a basic property of restrained groups in Lemma 3, leading to the proof of Theorem A followed by the Lemma 7 and the proof of Theorem B.

Lemma 1. A group $G$ is strongly restrained if

(i) it is an $n$-Engel group for some $n \geq 1$, or

(ii) it is $n$-collapsing for some $n \geq 1$.

Proof. Let $x, y$ be elements in $G$. Then the subgroup generated by $\{x,[x, y]$ $\left.=[x, 1 y], \ldots,[x, i+1 y]=\left[x,{ }_{i} y, y\right], \ldots,[x, r y]\right\}$ is precisely the subgroup generated by $\left\{x, x^{y}, \ldots, x^{y^{r}}\right\}$ as is easily seen by inducting on $r$. Thus if $G$ is an $n$-Engle group, then $\left\langle x^{(y)}\right\rangle=\left\langle x, x^{y}, \ldots, x^{y^{n-1}}\right\rangle$.

(ii) Let $S=\left\{x y^{-1}, x y^{-2}, \ldots, x y^{-n}\right\}$. Then there exist two distinct functions $f, g$ on the set $\{1,2, \ldots, n\}$ such that

$$
\prod_{i=1, \ldots, n} x y^{f(i)}=\prod_{i=1, \ldots, n} x y^{g(i)}
$$

Let $r$ be the largest integer such that $f(r) \neq g(r)$, let $s(i)=f(1)+\cdots+f(i)$, and let $t(i)=g(1)+\cdots+g(i)$. Then we get the equality

$$
x x^{y^{-s(1)}} x^{y^{-s(2)}} \cdots x^{y^{-s(r-1)}} y^{s(r)}=x x^{y^{-t(1)}} x^{y^{-t(2)}} \cdots x^{y^{-t(r-1)}} y^{t(r)} .
$$

If $s(r) \neq t(r)$, then $y^{k} \in\left\langle x^{\langle y\rangle}\right\rangle$ for some $k>0$. Letting $m$ be the least positive integer such that $y^{m} \in\left\langle x^{\langle y\rangle}\right\rangle$, we get $\left\langle x^{\langle y\rangle}\right\rangle=\left\langle y^{m}, x^{y^{i}} ; 0 \leq i<m\right\rangle$. Note that $m<n^{2}$. If $s(r)=t(r)$ and $f(r) \neq g(r)$, then $s(r-1) \neq t(r-1)$, say, $s(r-1)<t(r-1)$. Then $x^{y^{s(r-1)}} \in\left\langle x, x^{y}, \ldots, x^{y^{s(r-1)-1}}\right\rangle$ and $\left\langle x^{\langle y\rangle}\right\rangle=$ $\left\langle x^{y^{i}} ;-s(r-1)<i<s(r-1)\right\rangle$, requiring fewer than $2 n^{2}$ generators.

Lemma 2. A group $G$ is restrained if it is an Engel group or if it is a collapsing group or if it satisfies the maximal condition for subgroups locally. 
Proof. The proof, in the first two cases, is identical to that of Lemma 1 and, in the last case, is trivial.

Lemma 3. Let $G$ be a finitely generated restrained group. If $H$ is a normal subgroup of $G$ such that $G / H$ is cyclic, then $H$ is finitely generated.

Proof. For some $g \in G$, we can write $G$ in the form $H\langle g\rangle$. Since $G$ is finitely generated, there exist $h_{1}, h_{2}, \ldots, h_{r}$ in $H$ such that $G=\left\langle h_{1}, h_{2}, \ldots, h_{r}, g\right\rangle$ and $H=\left\langle h_{1}, h_{2}, \ldots, h_{r}\right\rangle^{G}$. For each $i=1, \ldots, r,\left\langle h_{i}^{\langle g\rangle}\right\rangle$ is finitely generated, say, $\left\langle h_{i}^{\langle g\rangle}\right\rangle=\left\langle h_{i 1}, h_{i 2}, \ldots, h_{i d(i)}\right\rangle$. Now let $H_{1}=\left\langle h_{i l(i)} ; 1 \leq i \leq r, 1 \leq\right.$ $l(i) \leq d(i)\rangle$. Then clearly $g$ lies in $N_{G}\left(H_{1}\right)$, the normalizer of $H_{1}$ in $G$, and $\left\langle h_{1}, \ldots, h_{r}\right\rangle \leq H_{1}$. Hence $N_{G}\left(H_{1}\right)=G$. This means that $H_{1}=H$ and $H$ is finitely generated.

Corollary 4. Let $G$ be a finitely generated restrained group. Then $G^{\prime}$ is finitely generated.

This result follows readily from the repeated use of Lemma 3. In particular, if $G$ is a finitely generated soluble restrained group, then $G$ is polycyclic. Using Tit's Alternative [6] it also follows that a finitely generated restrained linear group is polycyclic-by-finite.

Corollary 5. A finitely generated residually finite strongly restrained group $G$ is polycyclic-by-finite.

Proof. If $G$ is $n$-restrained, then it has no section isomorphic to a twisted wreath product $E t w r_{L} H$ with $[H: L]>n$. Thus by Theorem 4 in [7], $G$ has a soluble subgroup of finite index and hence $G$ is polycyclic-by-finite.

Proof of Theorem A. If $H$ is polycyclic and $r$ is the length of a series from 1 to $H$ with cyclic factors, then every subgroup $H$ can be generated by $r$ elements as can be seen via induction on $r$. Now if $H \triangleleft G$ and $G / H$ is of order $s$, then every subgroup of $G$ can be generated by $s+r$ elements and $G$ is strongly restrained. That a polycyclic-by-finite group $G$ is residually finite is well known. Thus we have shown one way implication of Theorem $A$.

Now let $G$ be a finitely generated strongly restrained locally graded group. Let $R$ be the finite residual of $G$. Then $G / R$ is polycyclic-by-finite. Thus by repeated application of Corollary $4, R$ is also finitely generated. If $R \neq 1$, then it has a proper characteristic subgroup $K$ of finite index and $G / K$ is polycyclicby-finite so that $G / K$ is residually finite. Hence $R \subseteq K$, a contradiction.

We mention a few consequences of Theorem $A$. The first is a marginal improvement of the corresponding result in [7].

Corollary 6. If $G$ is an $n$-Engel locally graded group, then $G$ is locally nilpotent. Proof. It follows from Lemma 1 and Theorem A that $G$ is locally polycyclicby-finite. But polycyclic-by-finite Engel groups are nilpotent. Hence the result follows.

It is interesting to note that if the group $G$ in Corollary 6 is torsion-free, then it is nilpotent of class depending only on $n$, independent of the number of generators of $G$, as shown by Zalmanov in [8]. In particular a locally indicable $n$-Engel group is nilpotent. It also follows from Lemma 1 and Theorem A that if $G$ is a finitely generated collapsing locally graded group, then $G$ is 
polycyclic-by-finite. This provides a major reduction towards showing that $G$ is nilpotent-by-finite which was shown by Semple and Shalev in [5].

Lemma 7. If $G$ is a collapsing right-orderable group, then every right order on $G$ is a $C$-order.

Proof. Let $P$ be the positive cone of a given right order on $G$ and $a, b$ in $P$. Suppose, if possible, that $a^{m} b<a$ for all integers $m>0$. Consider the set $S=\left\{b a, b a^{2}, \ldots, b a^{n}\right\}$ where $n$ is such that $\left|S^{n}\right|<n^{n}$. Since $G$ is $n$-collapsing, there exist two distinct functions $f, g$ on the set $\{1,2, \ldots, n\}$ such that

$$
\prod_{i=1, \ldots, n} b a^{f(i)}=\prod_{i=1, \ldots, n} b a^{g(i)} .
$$

Hence for some $0<r \leq n$ we have $b a^{f(1)} b a^{f(2)} \cdots b a^{f(r)}=b a^{g(1)} b a^{g(2)} \cdots b a^{g(r)}$ and $f(r) \neq g(r)$. Say $f(r)<g(r)$, and let $s=g(r)-f(r)$. Then we have $b a^{f(1)} \ldots b=b a^{g(1)} \ldots b a^{s}$. Now $a^{m} b<a$ for all $m>0$ implies $b a^{f(1)} b a^{f(2)} \cdots b<a^{f(1)+1} b a^{f(2)} \cdots b<a^{f(2)+1} b \cdots b<\cdots<a$. On the other hand, $b a^{g(1)} \cdots b \geq e$ so that $b a^{g(1)} \cdots b a^{s} \geq a^{s} \geq a$, giving the required contradiction.

Proof of Theorem B. Let $G$ be a finitely generated collapsing $R O$-group. By Lemma 7 it has a $C$-order so that it is locally indicable (see [2]). By Lemma 3 and Theorem A, $G$ is polycyclic-by-finite. By Theorem in [5], $G$ is nilpotentby-finite.

Note that even the simplest example of the group $G=\left\langle a, b ; a^{b}=a^{-1}\right\rangle$ shows that a collapsing $R O$-group need not be nilpotent.

\section{REFERENCES}

1. R. Botto Mura and A. H. Rhemtulla, Orderable groups, Dekker, New York, 1977.

2. P. F. Conrad, Right-ordered groups, Michigan Math. J. 6 (1959), 267-275.

3. N. Gupta and S. Sidki, Some infinite p-groups, Algebra i Logika 22 (1983), 584-589.

4. A. Yu. Ol'shanskii, An infinite group with subgroups of prime orders, Math. USSR-Izv. 16 2 (1981), 279-289.

5. J. F. Semple and A. Shalev, Combinatorial conditions in residually finite groups I, J. Algebra (to appear).

6. J. Tits, Free subgroups in linear groups, J. Algebra 20 (1972), 250-270.

7. J. S. Wilson, Two-generator conditions for residually finite groups, Bull. London Math. Soc. 23 (1991), 239-248.

8. E. I. Zelmanov, On some problems of group theory and Lie algebras, Math. USSR-Sb. 66 (1990), 159-168.

Department of Mathematics, University of Alberta, Edmonton, Alberta, Canada T6G 2G1

E-mail address: akbar@malindi.math.ualberta.ca 